TITLE:

\title{
Analysis method for investigating the influence of mechanical components on dynamic mechanical error of machine tools
}

\section{$\operatorname{AUTHOR}(S)$ :}

Kono, Daisuke; Matsubara, Atsushi; Nagaoka, Kotaro; Yamazaki, Kazuo

\section{CITATION:}

Kono, Daisuke ... [et al]. Analysis method for investigating the influence of mechanical components on dynamic mechanical error of machine tools. Precision Engineering 2012, 36(3): 477-484

\section{ISSUE DATE:}

2012-07

URL:

http://hdl.handle.net/2433/155807

\section{RIGHT:}

(C) 2012 Elsevier Inc.; This is not the published version. Please cite only the published version.; この論文は出版社版でありません。引用の際に は出版社版をご確認ご利用ください。 


\title{
Analysis method for investigating the influence of mechanical components on dynamic mechanical error of machine tools
}

\author{
Daisuke Kono $^{1}$, Atsushi Matsubara ${ }^{1}$, Kotaro Nagaoka $^{2}$, Kazuo Yamazaki $^{3}$ \\ ${ }^{1}$ Dept. of Micro Engineering, Graduate School of Engineering, Kyoto University \\ Yoshida-honmachi, Sakyo-ku, Kyoto 606-8501, Japan \\ Email: kono@prec.kyoto-u.ac.jp \\ Phone: $+81-75-753-5226$ \\ Fax: $+81-75-753-5226$ \\ ${ }^{2}$ Advanced Technology R\&D Center, Mitsubishi Electric Corporation \\ 8-1-1 Tsukaguchi-honmachi, Amagasaki, Hyogo 661-8661, Japan \\ ${ }^{3}$ Department of Mechanical \& Aeronautical Engineering, University of California, \\ Davis, One Shields Avenue, Davis, CA95616, USA
}

\begin{abstract}
In machine tools, the difference between the position of the tool center point and that of position detectors of the control system leads to a dynamic mechanical error, which is obtained as the difference between the feedback-controlled table position and the position of the tool relative to the table (tool-table relative position). In this paper, analysis methods are proposed to roughly determine the component of the mechanical system that causes the dynamic mechanical error. Two methods, a two-encoders method and a four-accelerators method, for investigating the influence of the mechanical component on the dynamic mechanical error are proposed. In both methods, the frequency response function between the feedback-controlled table position and the tool-table relative position is evaluated. By the proposed methods, the dynamic mechanical error of a high-precision machining center in the $\mathrm{X}$ and $\mathrm{Y}$ directions is analyzed for frequencies up to $200 \mathrm{~Hz}$. It was found that the entire frequency range could be divided into three distinct subranges depending on how the component of the mechanical system influences the dynamic mechanical error at different frequencies. The analysis results indicated that in the low-frequency range, the dynamic response of the driven component plays a dominant role in influencing the dynamic mechanical error. Then, the dynamic mechanical error of the experimental machine was measured for small circular motions. The dynamic mechanical error occurred at the micrometer level. The dynamic mechanical error can be estimated from the frequency response function measured by the proposed method.
\end{abstract}

Keywords: dynamic mechanical error, dynamic response, high-precision machine tools, analysis method 


\section{Introduction}

In recent times, demands for high-precision machining that can achieve a geometric accuracy of submicrometer level are increasing to produce high-precision dies and molds for optical parts. High-precision machining requires not only accuracy but also productivity. Therefore, dynamic contouring errors of machine tools must be suppressed under high-speed conditions. Linear motor drives are widely used to enhance the response and reduce the motion error due to friction [1-3]. The dynamic response of the control system and the mechanical system also influences the dynamic contouring error under high-speed conditions. In particular, the dynamic response of the mechanical system causes the dynamic mechanical error which is defined as the difference between the position of the tool relative to the work table and the table position measured by position detectors of the control system.

A number of control schemes have been proposed to reduce the dynamic contouring error in multi-axes motion. Methods for tuning control parameters have been used to match the response to commands among all axes [4]. Model-reference feedforward (MR-FF) controllers are also used so that the feedforward and feedback controller can be designed independently to match the dynamic response $[5,6]$. The cross-coupled control (CCC) method proposed by Koren et al. is a popular method to compensate contouring errors [7-9]. In CCC, the error is calculated from the deviation of each axis. The above approaches are effective in reducing the dynamic contouring error at the position detector.

However, to date, few studies have investigated the dynamic mechanical error of machine tools. Franse et al. experimentally evaluated the dynamic response of an ultraprecision machine tool to external disturbance forces [10]. Pereira et al. measured 
the dynamic mechanical error of a coordinate measuring machine performing circular probe motions [11]. Although the error is modeled as a function of normal acceleration in their study, the error model is obtained by fitting measurement data. The influence of the mechanical system on the error is not explained clearly.

Modal analysis is effective to analyze the dynamic response of the mechanical system and determine the cause of an undesirable relative position between two components. However, it is practically difficult to determine the cause of the dynamic mechanical error because the error is influenced by two relative positions.

In this study, analysis methods are proposed to roughly determine the component of the mechanical system that causes the dynamic mechanical error. In this analysis, the dynamic mechanical error is also estimated from the table position measured by position detectors of the control system. The dynamic mechanical error of a high-precision machining center is analyzed with the proposed method. Then, the dynamic mechanical error of the machining center for circular motions is measured to compare the measured error and the error estimated with the proposed method.

\section{Method for analyzing dynamic mechanical error}

\subsection{Concept of the method}

Figure 1 shows the schematic of a machine tool during table motion. The table position is detected and controlled with a linear encoder. In Fig.1, it is assumed that the scanning head of the linear encoder is attached to the driven component of the machine and the scale of the linear encoder is fixed on the fixed component. During table motion phase, if the position of the linear encoder differs from the tool center position, a difference will occur between the position of the tool relative to the table and the table 
position measured by the linear encoder because of the dynamic response of the mechanical system to the driving force and counter force. In this paper, this difference is defined as the dynamic mechanical error. Henceforth, the position of the tool relative to the table is referred to as a T-T (Tool-Table) relative position. The table position measured by the linear encoders is referred to as a feedback-controlled table position.

The mechanical system of the machine tool should be modified to reduce the dynamic mechanical error. Modal analysis is effective to analyze the dynamic response of the mechanical system and determine the cause of an undesirable relative position (such as a relative vibration) between two components. However, because the dynamic mechanical error is influenced by two relative positions, it is difficult to determine which relative position causes the error.

In this paper, two analysis methods are proposed to roughly determine the cause of the dynamic mechanical error. One method is referred to as a two-encoders method (2E method) and the other is referred to as a four-accelerometers method (4A method). In both methods, the frequency response function $G_{e t}(s)$ between the feedback-controlled table position and the T-T relative position is obtained to evaluate the dynamic mechanical error. The frequency response function $G_{\text {ferel }}(s)$ between the driving force and the feedback-controlled table position and the frequency response function $G_{f t r e l}(s)$ between the driving force and the T-T relative position are measured to investigate which response influences $G_{e t}(s)$. Once the cause of the dynamic mechanical error is determined by the proposed method, the modal analysis can be used to decide the component to be modified in detail. The function $G_{e t}(s)$ can be also used to estimate the dynamic mechanical error from the feedback-controlled table position. The details of these methods are as follows. 


\subsection{Two-encoders method}

The function $G_{e t}(s)$ is obtained by the following equation:

$$
G_{e t}(s)=\frac{G_{\text {frrel }}(s)}{G_{\text {ferel }}(s)}
$$

To obtain the functions $G_{f e r e l}(s)$ and $G_{f t r e l}(s)$, the feedback-controlled table position and the $\mathrm{T}-\mathrm{T}$ relative position are measured with the linear encoder and a $2 \mathrm{D}$ grid encoder (such as KGM, HEIDENHAIN). The driving force can be calculated from the motor current feedback and the force constant of the drive system.

The dynamic mechanical error is directly measured by the $2 \mathrm{E}$ method. Compared to the $4 \mathrm{~A}$ method, the $2 \mathrm{E}$ method can achieve higher resolution in position measurement and higher sensitivity in the low-frequency range of 0 -several Hertz.

\subsection{Four-accelerometers method}

The function $G_{e t}(s)$ is obtained by the following equation:

$$
G_{e t}(s)=\frac{G_{\text {frrel }}(s)}{G_{\text {ferel }}(s)}=\frac{G_{\text {ftool }}(s)-G_{\text {ftable }}(s)}{G_{\text {fscale }}(s)-G_{\text {fhead }}(s)}
$$

where $G_{\text {ftool }}(s), G_{\text {ftable }}(s), G_{\text {fhead }}(s)$, and $G_{\text {fscale }}(s)$ are frequency response functions between the driving force and the absolute positions of the tool tip, the table, the scanning head of the linear encoder, and the scale of the linear encoder, respectively. Each frequency response function is measured with an accelerometer. The driving force can be obtained as described in Section 2.2.

Compared to the $2 \mathrm{E}$ method, the cause of the dynamic mechanical error can be determined more clearly in the $4 \mathrm{~A}$ method because the influence of the dynamic responses of the fixed and driven component on $G_{f e r e l}(s)$ and $G_{f t r e l}(s)$ can be analyzed. 
However, the sensitivity of the accelerometers in the low-frequency range limits the bandwidth of the measurement.

\section{Analysis of dynamic mechanical error of a machine tool}

\subsection{Machine tool used in the experiment}

The dynamic mechanical error of a machine tool is analyzed by the proposed methods. Figure 2 shows a photograph of the experimental machine. Its major specifications are listed in Table 1. The machine has three linear axes and is designed to achieve a motion accuracy of submicrometer level under high-speed conditions. The machine employs linear motor drives and high-precision rolling guideways. The $\mathrm{Y}$ and $\mathrm{Z}$ axes have twin drive units to avoid yaw. The $\mathrm{Y}$ axis is driven by the $\mathrm{Y}_{1}$ and $\mathrm{Y}_{2}$ drives, and the $\mathrm{Z}$ axis is driven by the $\mathrm{Z}_{1}$ and $\mathrm{Z}_{2}$ drives.

The feedback-controlled table position is measured by linear encoders with a resolution of $0.1 \mathrm{~nm}$. The feedback-controlled table position in the $\mathrm{Y}$ direction is measured by the linear encoder of the $\mathrm{Y}_{1}$ drive. A 2D grid encoder with a resolution of 1 $\mathrm{nm}$ (KGM182, HEIDENHAIN [12]) is installed to measure the T-T relative position in the $\mathrm{X}$ and $\mathrm{Y}$ directions. A grid plate of the 2D grid encoder is mounted on the table and a scanning head of the $2 \mathrm{D}$ grid encoder is attached to the spindle in place of a tool. The specifications of these measuring instruments are listed in Table 2.

A commercial NC system is used to control the drives. The NC servo system uses PI control in the motor current loop, PI control in the velocity loop and P control in the position loop. The servo system also has a MR-FF controller to design the response of the whole control system and the feedback system independently [5]. With the MR-FF controller, the synchronization error among axes is eliminated and the motion error due 
to external disturbances is suppressed. The bandwidth of the velocity loops of $\mathrm{X}$ and $\mathrm{Y}$ axes is about $170 \mathrm{~Hz}$ and $110 \mathrm{~Hz}$, respectively. The gain of the reference model is set to $300 \mathrm{rad} / \mathrm{s}$.

\subsection{Experimental method}

In this experiment, the dynamic mechanical error is analyzed by the 4A method. The 2E method is also used to verify $G_{e t}(s)$. In both methods, the machine is excited in the X and $\mathrm{Y}$ directions independently by drive units to obtain the frequency response functions. An analog chirp signal is input to the servo amplifier as the velocity command of drive units.

Figure 3(a) illustrates the experimental setup in the 2E method. While the machine is excited, the feedback-controlled table position, the T-T relative position, and the motor current feedback are measured using a synchronized data acquisition system [13]. The acquired data are input to the PC and $G_{f e r e l}(s)$ and $G_{f t r e l}(s)$ are calculated. The driving force is obtained by multiplying the motor current feedback and the torque constant of the motor.

Figure 3(b) shows the experimental setup in the 4A method. Two accelerometers are attached to the table and the scanning head of the $2 \mathrm{D}$ grid encoder to measure $G_{f t a b l e}(s)$ and $G_{f t o o l}(s)$, respectively. The location of the other two accelerometers is shown in Fig.4. According to the assumption described in Section 2.1, $G_{f h e a d}(s)$ and $G_{f s c a l e}(s)$ are measured at the linear encoder scale and the linear encoder scanning head, respectively, in the measurement in the $\mathrm{X}$ direction (Fig.4(a)). In the measurement in the Y direction, $G_{\text {fhead }}(s)$ and $G_{\text {fscale }}(s)$ are measured at the linear encoder scanning head and the linear encoder scale of the $\mathrm{Y}_{1}$ drive, respectively (Fig.4(b)). The frequency response functions 
are obtained using a FFT analyzer. The driving force is obtained in the same manner as the $2 \mathrm{E}$ method.

In both methods, the measurement is conducted for frequencies up to $200 \mathrm{~Hz}$. The frequency range is divided into three subranges of $0.2-20 \mathrm{~Hz}, 10-100 \mathrm{~Hz}$, and $50-200$ $\mathrm{Hz}$ to adjust the excitation force. In each frequency range, the machine is excited for 90 $\mathrm{s}$ and the measured data are averaged. The number of sample points is set to 1024 .

\subsection{Comparison of four-accelerometers method and two-encoders method}

Figures 5 and 6 show $G_{e t}(s)$ in the $\mathrm{X}$ and $\mathrm{Y}$ directions, respectively. In both figures, $G_{e t}(s)$ obtained by the $4 \mathrm{~A}$ method is close to the $G_{e t}(s)$ obtained by the $2 \mathrm{E}$ method. For frequencies up to $5 \mathrm{~Hz}$, the $G_{e t}(s)$ obtained by the $4 \mathrm{~A}$ method has greater noise.

Ideally, the magnitude and phase of $G_{e t}(s)$ are $0 \mathrm{~dB}$ and $0 \mathrm{deg}$., respectively. However, several peaks and valleys are observed in the magnitude plot in Figs.5 and 6. The magnitude increases over the entire frequency range in the $\mathrm{Y}$ direction, which is not seen in the $\mathrm{X}$ direction. This difference between $G_{e t}(s)$ in the $\mathrm{X}$ and $\mathrm{Y}$ directions is discussed in Section 3.5.

\subsection{Analyses in the $\mathrm{X}$ and $\mathrm{Y}$ directions}

\subsubsection{Analysis in the $\mathrm{X}$ direction}

Figure 7 shows $G_{\text {ferel }}(s)$ and $G_{\text {ftrel }}(s)$ obtained by the 4 A method. For frequencies higher than $100 \mathrm{~Hz}$, the peak-valley patterns of the magnitude of $G_{\text {ferel }}(s)$ and $G_{\text {ftrel }}(s)$ are different. This difference causes peaks and valleys of $G_{e t}(s)$, as shown in Fig.5. In the low-frequency range, the magnitude of $G_{\text {ftrel }}(s)$ is slightly larger than that of $G_{\text {ferel }}(s)$.

Then, the influence of $G_{f t o o l}(s)$ and $G_{f t a b l e}(s)$ on $G_{f t r e l}(s)$ is discussed. Figure 8 shows 
$G_{f t o o l}(s)$ and $G_{f t a b l e}(s)$. The function $G_{f t a b l e}(s)$ has no resonance peak in the magnitude plot and is dominated by the mass of the driven component. On the other hand, several resonances and antiresonances, which represent structural vibration modes of the fixed component, are seen in $G_{\text {ftool }}(s)$. The peak at $30 \mathrm{~Hz}$ corresponds to the rocking mode about the $\mathrm{Y}$ axis.

The magnitude of $G_{f t a b l e}(s)$ is larger than that of $G_{f t o o l}(s)$ over the entire frequency range and $G_{f t r e l}(s)$ is obtained by subtracting $G_{f t a b l e}(s)$ from $G_{f t o o l}(s)$. Therefore, the larger the difference in magnitude becomes, the larger the influence of $G_{f t a b l e}(s)$ on $G_{f t r e l}(s)$ becomes. Then, the entire frequency range can be divided according to the influence of $G_{f t a b l e}(s)$ and $G_{f t o o l}(s)$ on $G_{f t r e l}(s)$ into the following three subranges.

(1) 0-25 Hz: For frequencies lower than the natural frequency of the lowest structural vibration mode, the influence of $G_{f t a b l e}(s)$ is dominant because the magnitude of $G_{f t a b l e}(s)$ is more than $20 \mathrm{~dB}$ larger than that of $G_{f t o o l}(s)$.

(2) $25-100 \mathrm{~Hz}$ : The influence of $G_{f t a b l e}(s)$ is basically dominant. The function $G_{f t o o l}(s)$ also has an influence around its resonance frequency because the difference in the magnitude is about $10 \mathrm{~dB}$ and not large enough.

(3) 100-200 Hz: Both $G_{f t a b l e}(s)$ and $G_{f t o o l}(s)$ have an influence.

Figure 9 shows $G_{\text {fhead }}(s)$ and $G_{f s c a l e}(s)$. The influence of $G_{\text {fhead }}(s)$ and $G_{\text {fscale }}(s)$ on $G_{\text {ferel }}(s)$ can also be discussed as described above. Therefore, it is concluded that $G_{e t}(s)$ in the low frequency range is dominated by the dynamic response of the driven component. 


\subsubsection{Analysis in the $\mathrm{Y}$ direction}

Figure 10 shows $G_{f e r e l}(s)$ and $G_{f t r e l}(s)$ in the Y direction obtained by the $4 \mathrm{~A}$ method. The magnitude of $G_{f t r e l}(s)$ is larger than that of $G_{\text {ferel }}(s)$. For frequencies higher than 100 $\mathrm{Hz}$, the magnitude difference increases with the increase of the frequency, which results in the increasing magnitude of $G_{e t}(s)$ (as shown in Fig.6). The influence of $G_{f t o o l}(s), G_{\text {ftable }}(s), G_{\text {fhead }}(s)$, and $G_{\text {fscale }}(s)$ on $G_{e t}(s)$ can also be explained as described in Section 3.4.1.

\subsection{Analysis of the difference between the $\mathrm{X}$ and $\mathrm{Y}$ directions}

As described in Section 3.3, the magnitude of $G_{e t}(s)$ increases over the entire frequency range in the $\mathrm{Y}$ direction, which is not seen in the $\mathrm{X}$ direction. This difference between $G_{e t}(s)$ in the $\mathrm{X}$ and $\mathrm{Y}$ directions is caused by the magnitude difference between $G_{\text {ferel }}(s)$ and $G_{f t r e l}(s)$ in each direction.

The Nyquist plots of $G_{f t o o l}(s), G_{f t a b l e}(s), G_{f h e a d}(s)$, and $G_{f s c a l e}(s)$ in the Y direction are shown in Fig.11 to discuss the magnitude difference between $G_{f e r e l}(s)$ and $G_{f t r e l}(s)$. The one in the $\mathrm{X}$ direction is also shown in Fig.12. In Figs.11 and 12, the response for frequencies higher than $100 \mathrm{~Hz}$ is plotted and the color of markers indicates the variation of the frequency. Responses $G_{\text {ferel }}(s)$ and $G_{f t r e l}(s)$ are obtained as vectors between the plotted responses. In Fig.11, it is obviously seen that the magnitude difference between $G_{f e r e l}(s)$ and $G_{f t r e l}(s)$ increases with the increase of the frequency. On the other hand, in Fig.12, the magnitude of $G_{f t r e l}(s)$ decreases by the phase delay of $G_{f t o o l}(s)$ between 100 and $160 \mathrm{~Hz}$, and the resultant magnitude difference between $G_{\text {ferel }}(s)$ and $G_{\text {ftrel }}(s)$ is small. This phase delay is caused by the vibration mode in the X direction between the bed and the driven component of the $\mathrm{Y}$ axis. 


\section{Measurement of dynamic mechanical error for circular motions}

The dynamic mechanical error of the experimental machine is measured for practical motions. Measured error is compared to $G_{e t}(s)$ to verify the analysis with the proposed method.

\subsection{Measurement method}

For circular motions in the XY plane, the T-T relative position and the feedback-controlled table position are measured simultaneously to obtain the dynamic mechanical error. The measurement system shown in Fig.3(a) is used also in this experiment.

The measurement is carried out for clockwise circular motions. The experimental conditions for circular motions are summarized in Table 3 . The circular motion is continued for several rotations to conduct the measurement under steady state conditions. The number of rotations is four for $1 \mathrm{~mm}$ radius and two for other radii. The sampling time is set to $1.8 \mathrm{~ms}$.

\subsection{Measurement result}

The error contours at $5 \mathrm{~m} / \mathrm{min}$ are shown in Fig.13. In Fig.13 (b) and (c), the difference is observed in the error contours around the start and end of motion because of the transient response of the machine. When the radius is $5 \mathrm{~mm}$, the dynamic mechanical error is less than $1 \mu \mathrm{m}$. However, as the radius decreases, the dynamic mechanical error increases and reaches to about $4 \mu \mathrm{m}$. In Fig.13(c), the elliptical distortion is observed in the T-T relative position and the feedback-controlled table position, which is caused by the difference between phase delays of the $\mathrm{X}$ and $\mathrm{Y}$ drives. 
The experimental result shown in Fig.13 shows that the diameter of the T-T relative position is larger than that of the feedback-controlled table position. The diameter ratio in the $\mathrm{X}$ and $\mathrm{Y}$ directions between the $\mathrm{T}-\mathrm{T}$ relative position and the feedback-controlled table position should correspond to the magnitude of $G_{e t}(s)$.

Figures 14 shows the comparison between the magnitude of $G_{e t}(s)$ measured in Chapter 3 by the $2 \mathrm{E}$ method and the diameter ratio in the $\mathrm{X}$ and $\mathrm{Y}$ directions. In both directions, although spike noises are observed in $G_{e t}(s)$, the magnitude of $G_{e t}(s)$ is larger than $0 \mathrm{~dB}$ even in the low-frequency range and is positively correlated with the frequency.

It can be seen that the variation of the diameter ratio corresponds to the magnitude of $G_{e t}(s)$. Ignoring the spike noises, the difference between the diameter ratio and the magnitude of $G_{e t}(s)$ is about $0.01 \mathrm{~dB}$ at maximum. This difference corresponds to the dynamic mechanical error of $1.2 \mu \mathrm{m}$ under the condition 6 shown in Table 3 . The results in Fig. 14 indicate that the dynamic mechanical for small circular motions can be estimated by $G_{e t}(s)$.

\section{Conclusions}

Two methods were proposed to roughly determine the component of the mechanical system that causes the dynamic mechanical error. By the proposed methods, the dynamic mechanical error of a high-precision machining center was analyzed in the $\mathrm{X}$ and Y directions for frequencies up to $200 \mathrm{~Hz}$. The dynamic mechanical error of the experimental machine was measured for small circular motions. Measured error is compared to the dynamic response of the machine to verify the analysis with the proposed method. From this study, the following conclusions have been drawn. 
(1) The two-encoders method and the four-accelerometers method were proposed for investigating the influence of the mechanical component on the dynamic mechanical error. In both methods, the frequency response function $G_{e t}(s)$ between the feedback-controlled table position and the tool-table relative position is evaluated.

(2) By the four-accelerometers method, influences of the driven and fixed components of the machine were separately investigated. It was found that the entire frequency range could be divided into three distinct subranges depending on how the component of the mechanical system influences the dynamic mechanical error at different frequencies. The analysis results indicated that in the low-frequency range, the dynamic response of the driven component plays a dominant role in influencing the dynamic mechanical error.

(3) The dynamic mechanical error occurred at the micrometer level for small circular motions. The measured dynamic mechanical error can be estimated by $G_{e t}(s)$.

\section{Acknowledgement}

This research is carried out in MTTRF-KRI (Machine Tool Technologies Research Foundation, Kyoto Research Institute) project. The project is supported by Mori Seiki Co., Ltd., THK Co., Ltd., Mitsubishi Electric Corporation, Tokkyokiki Corporation, and Magnescale Co., Ltd.. The authors wish to thank their help. 


\section{References}

[1] Z.G. Wang, X.Cheng, K.Nakamoto, S.Kobayashi and K.Yamazaki: Design and development of a precision machine tool using counter motion mechanisms, International Journal of Machine Tools \& Manufacture, 2010, Vol.50, 357.

[2] Hidenori Shinno, Hitoshi Hashizume: Nanometer Positioning of a Linear Motor-Driven Ultraprecision Aerostatic Table System with Electrorheological Fluid Dampers, Annals of the CIRP, 1999, Vol.48, 289.

[3] Chuan Yang, Zhi Zhang, Qiang Zhao: The study on precision positioning system of two-dimensional platform based on high speed and large range, Precision Engineering, 2010, Vol.34, 640.

[4] Xue-Cheng Xi, Aun-Neow Poo, Geok-Soon Hong: Improving contouring accuracy by tuning gains for a bi-axial CNC machine, International Journal of Machine Tools \& Manufacture, 2009, Vol.49, 395.

[5] Kotaro Nagaoka and Tomonori Sato: Feedforward Controller for Continuous Path Control of CNC Machine Tools, International Journal of Simulation, 2006, Vol.7, 39 .

[6] Iwasaki T., Sato T., Morita A., and Maruyama H.: Auto-Tuning of Two-Degree-of-Freedom Motor Control for High-Accuracy Trajectory Motion. Control Engineering Practice, 1996, Vol.4, 537.

[7] Yoram Koren: Cross-coupled biaxial computer control for manufacturing systems, ASME Transactions, Journal of Dynamic Systems, Measurement and Control, 1980, Vol.102, 265-271.

[8] Jih-Hua Chintz: and Tsung-Ching Lin: Cross-Coupled Precompensation 
Method for the Contouring Accuracy of Computer Numerically Controlled Machine Tools, International Journal of Machine Tools \& Manufacture, 1997, Vol.37, 947-967.

[9] Ke-Han Su and Ming-Yang Cheng: Contouring accuracy improvement using cross-coupled control and position error compensator, International Journal of Machine Tools \& Manufacture, 2008, Vol.48, 1444-1453.

[10] J. Franse, J. W. Roblee,K. Modemann: Dynamic characteristics of the Lawrence Livermore National Laboratory Precision Engineering Research Lathe, Precision Engineering, 1991, Vol.13, 196-202.

[11] P.H. Pereira, R.J. Hocken: Characterization and compensation of dynamic errors of a scanning coordinate measuring machine, Precision Engineering, 2007, Vol.31, 22-32.

[12] Measuring systems for inspecting linear axes: KGM181 and KGM 182 grid encoders, Measuring systems for machine tool inspection and acceptance testing (The brochure of the Heidenhain), 2007, 10-11

[13] Kotaro Nagaoka, Atsushi Matsubara, Tomoya Fujita, and Tomonori Sato: Analysis Method of Motion Accuracy Using NC System with Synchronized Measurement of Tool-Tip Position, International Journal of Automation Technology, 2009, Vol.3, 394-400 


\section{List of Figure Captions}

Figure 1 Schematic of a machine tool during table motion.

Figure 2 Photograph of the machine tool used in the experiment.

Figure 3 Experimental setup for the analysis of dynamic mechanical error.

(a) Two-encoders method.

(b) Four-accelerometers method.

Figure 4 Location of accelerometers in the four-accelerometers method.

(a) Measurement in the $\mathrm{X}$ direction. Splash guards and the 2D grid plate are removed.

(b) Measurement in the $\mathrm{Y}$ direction.

Figure $5 G_{e t}(s)$ in the $\mathrm{X}$ direction measured by the four-accelerometers method and the two-encoders method.

Figure $6 G_{e t}(s)$ in the Y direction measured by the four-accelerometers method and the two-encoders method.

Figure $7 G_{\text {ferel }}(s)$ and $G_{\text {ftrel }}(s)$ in the $\mathrm{X}$ direction obtained by the four-accelerometers method.

Figure $8 G_{f t o o l}(s)$ and $G_{f t a b l e}(s)$ in the X direction.

Figure $9 G_{\text {fscale }}(s)$ and $G_{\text {fhead }}(s)$ in the X direction.

Figure $10 G_{\text {ferel }}(s)$ and $G_{\text {ftrel }}(s)$ in the Y direction obtained by the four-accelerometers method

Figure 11 Nyquist plot of $G_{f t o o l}(s), G_{f t a b l e}(s), G_{f s c a l e}(s)$, and $G_{f h e a d}(s)$ in the Y direction.

Figure 12 Nyquist plot of $G_{f t o o l}(s), G_{\text {ftable }}(s), G_{\text {fscale }}(s)$, and $G_{f h e a d}(s)$ in the X direction.

Figure 13 Error contours for circular motion. Feed rate $=5 \mathrm{~m} / \mathrm{min}$.

Figure 14 Comparison between the magnitude of $G_{e t}(s)$ and the diameter ratio.
(a) Comparison in the $\mathrm{X}$ direction.

(b) Comparison in the $\mathrm{Y}$ direction. 
Table 1 Specifications of the machine tool.

\begin{tabular}{|c|c|c|}
\hline \multirow{2}{*}{ Machine } & Size & $\mathrm{W} \times \mathrm{D} \times \mathrm{H}: 850 \mathrm{~mm} \times 1900 \mathrm{~mm} \times \mathrm{H}: 2200 \mathrm{~mm}$ \\
\hline & Mass & $2500 \mathrm{~kg}$ \\
\hline \multirow{7}{*}{$\begin{array}{l}\text { Drive } \\
\text { system }\end{array}$} & Mechanism & $\begin{array}{c}\text { Coreless linear motor } \\
\mathrm{X} \text { : single drive, } \mathrm{Y} \text { and } \mathrm{Z} \text { : twin drive }\end{array}$ \\
\hline & Driven mass & X: $60 \mathrm{~kg}, \mathrm{Y:} 220 \mathrm{~kg}, \mathrm{Z}: 220 \mathrm{~kg}$ \\
\hline & Travel distance & $\mathrm{X}: 130 \mathrm{~mm}, \mathrm{Y}: 225 \mathrm{~mm}, \mathrm{Z}: 150 \mathrm{~mm}$ \\
\hline & Guideway type & Linear rolling guideway \\
\hline & Maximum feed rate & $15 \mathrm{~m} / \mathrm{min}$ \\
\hline & Encoder resolution & $0.1 \mathrm{~nm}$ \\
\hline & $\begin{array}{l}\text { Maximum } \\
\text { acceleration }\end{array}$ & $2 \mathrm{G}$ \\
\hline
\end{tabular}


Table 2 Specifications of measuring instruments.

\begin{tabular}{lcc}
\hline & Resolution & $0.1 \mathrm{~nm}$ \\
Linear encoder & Accuracy & $\pm 2.5 \mu \mathrm{m} /$ full stroke \\
\hline 2D grid encoder & Resolution & $1 \mathrm{~nm}$ \\
& Accuracy & $\pm 2 \mu \mathrm{m} / 230 \mathrm{~mm}$ \\
\hline Accelerometer & Type & Piezoelectric \\
& Rensitivity & $50 \mathrm{mV} / \mathrm{m} / \mathrm{s}^{2}$ \\
& Bandwidth & $6 \times 10^{-4} \mathrm{~m} / \mathrm{s}^{2} \mathrm{rms}$ \\
& & $0.5-3000 \mathrm{~Hz}$ \\
\hline
\end{tabular}


Table 3 Experimental conditions for circular motion.

\begin{tabular}{ccccc}
\hline Condition & $\begin{array}{c}\text { Feed rate } \\
\mathrm{m} / \mathrm{min}\end{array}$ & $\begin{array}{c}\text { Radius } \\
\mathrm{mm}\end{array}$ & $\begin{array}{c}\text { Angular velocity } \\
\mathrm{rad} / \mathrm{s} \\
\text { (Frequency Hz) }\end{array}$ & $\begin{array}{c}\text { Number of } \\
\text { rotations }\end{array}$ \\
\hline 1 & 3 & 5 & $10(1.6)$ & 2 \\
2 & 3 & 2 & $25(4.0)$ & 2 \\
3 & 3 & 1 & $50(8.0)$ & 4 \\
4 & 5 & 5 & $16.7(2.7)$ & 2 \\
5 & 5 & 2 & $41.7(6.6)$ & 2 \\
6 & 5 & 1 & $83.3(13.3)$ & 4 \\
\hline
\end{tabular}




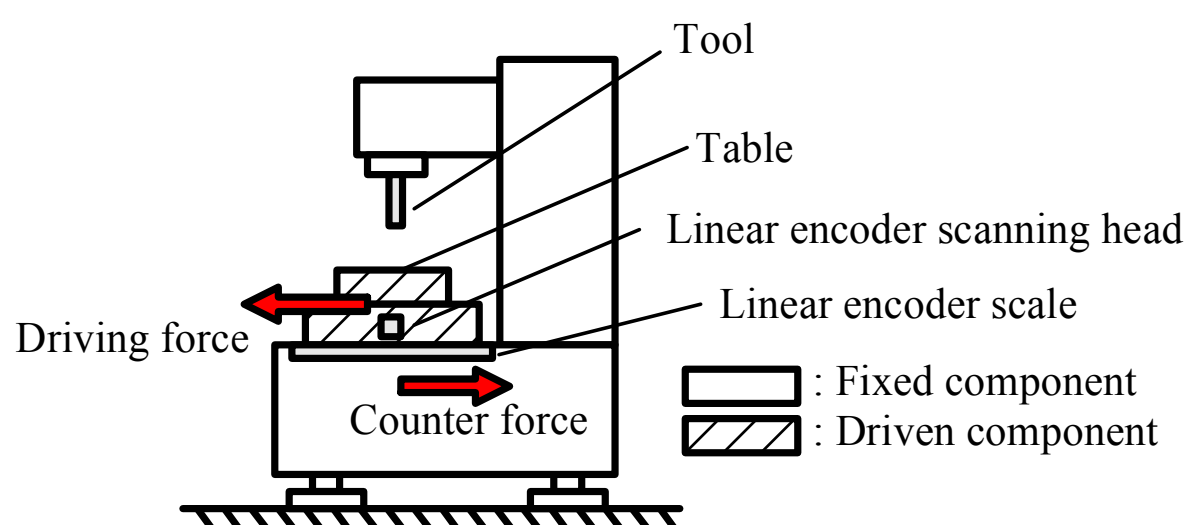

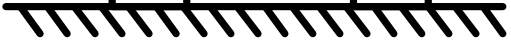

Fig. 1 Schematic of a machine tool during table motion. 


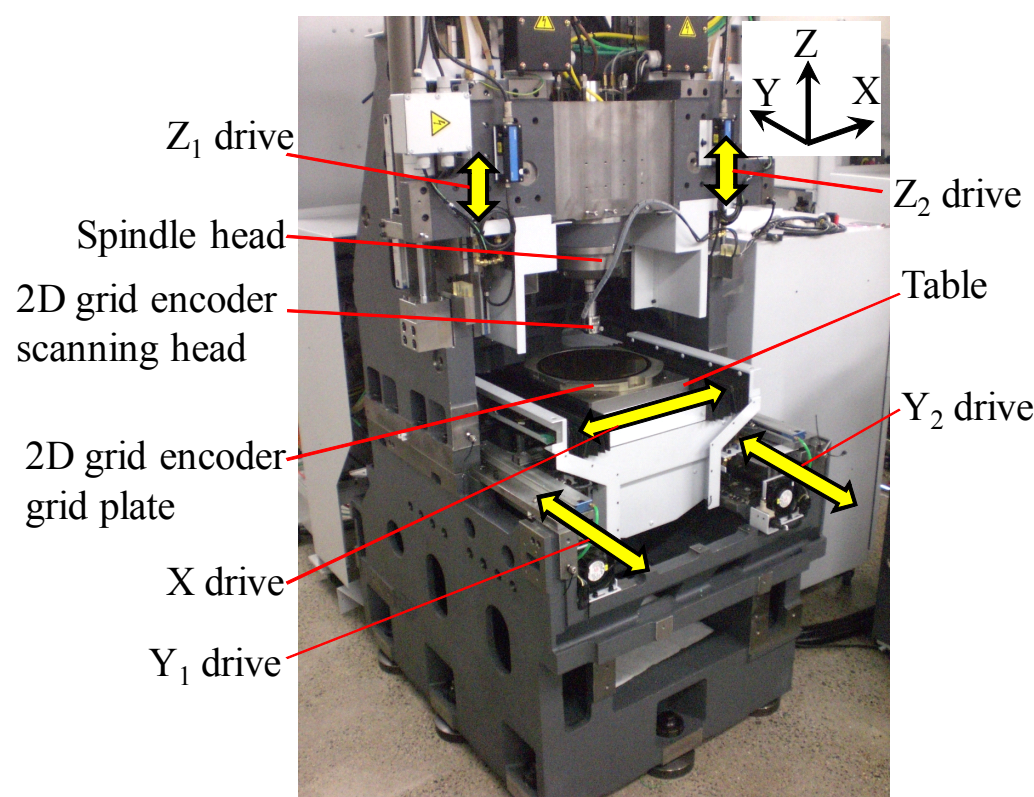

Fig.2 Photograph of the machine tool used in the experiment. 


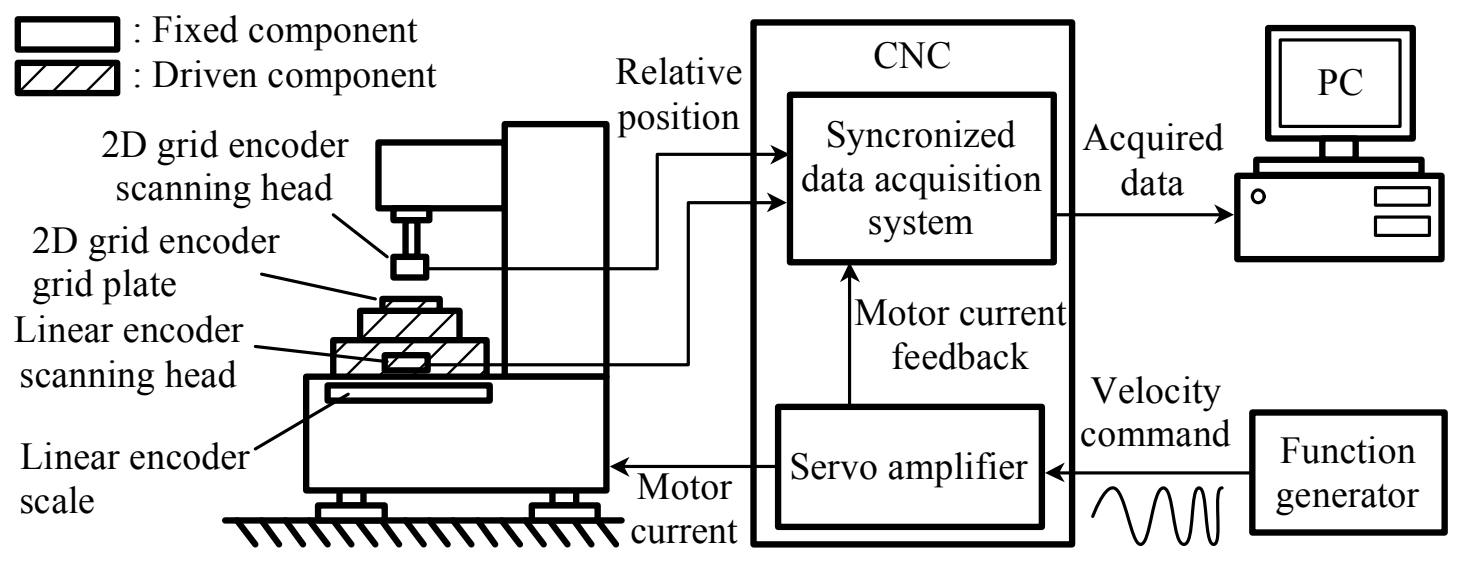

(a) Two-encoders method.

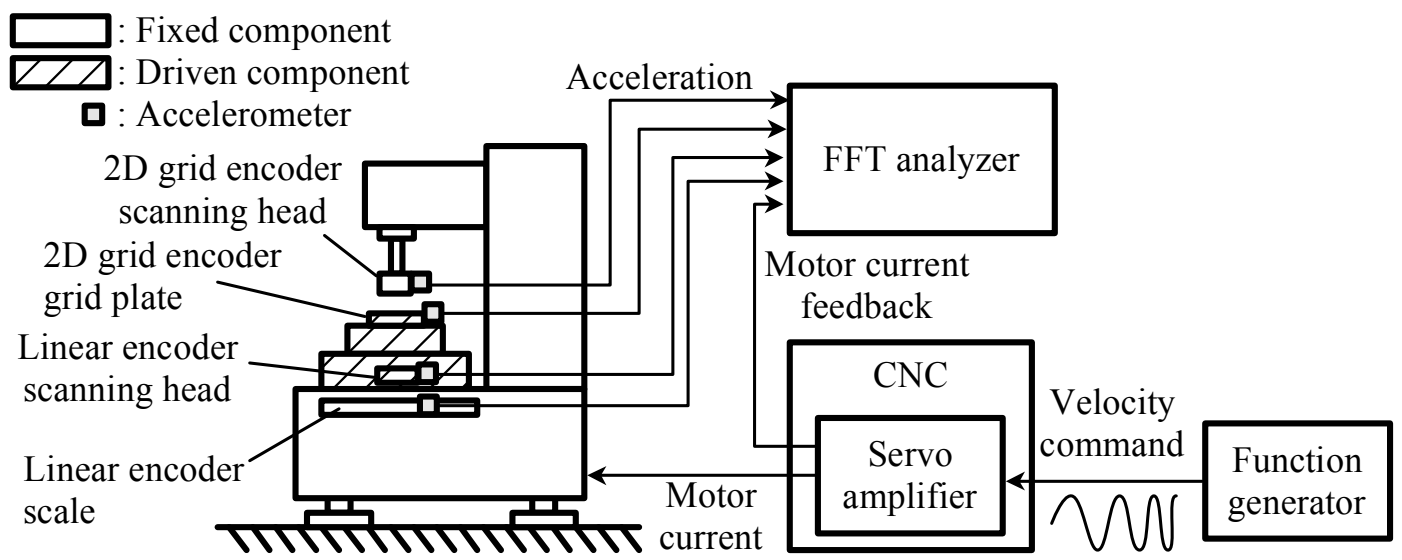

(b) Four-accelerometers method.

Fig. 3 Experimental setup for the analysis of dynamic mechanical error. 


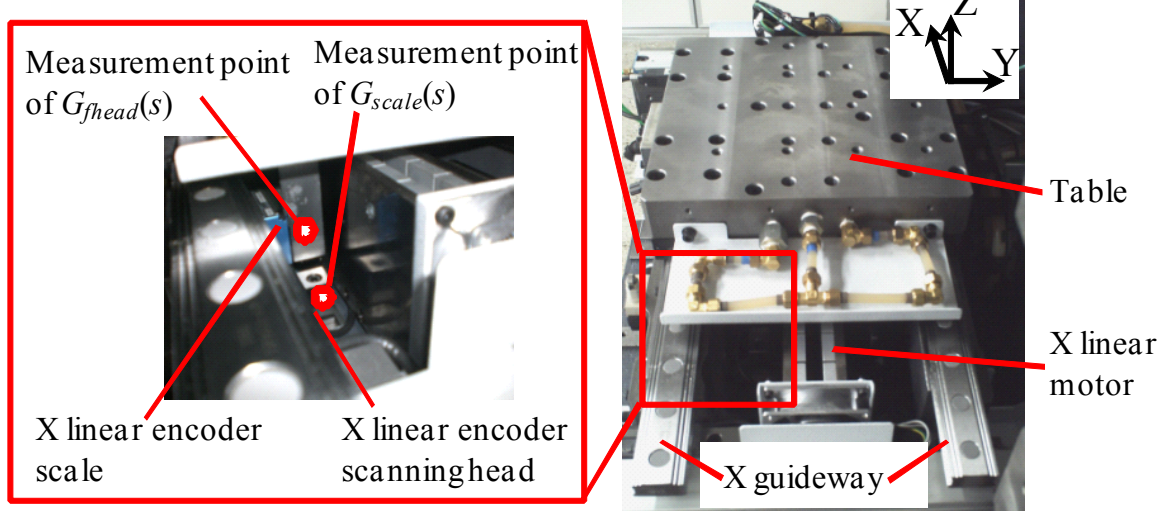

(a) Measurement in the $\mathrm{X}$ direction. Splash guards and the 2D grid plate are removed.

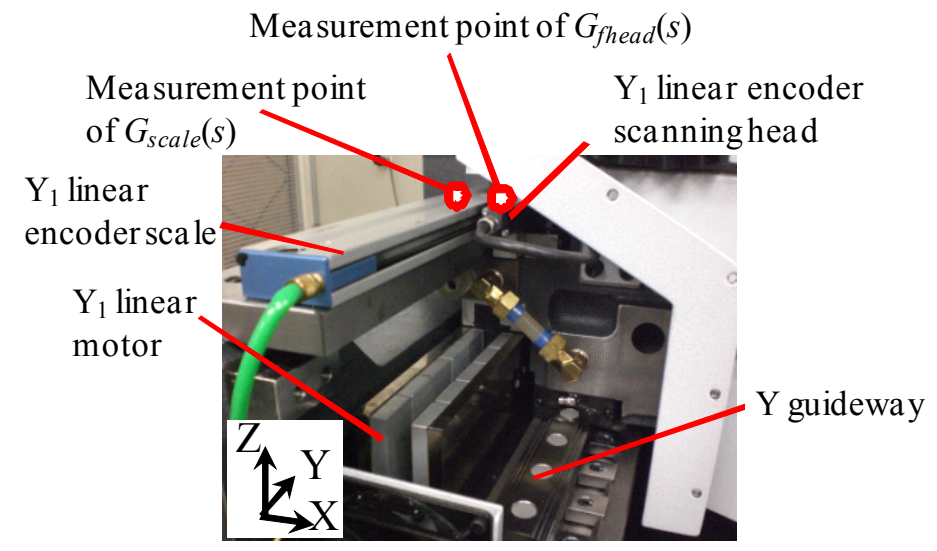

(b) Measurement in the Y direction.

Fig.4 Location of accelerometers in the four-accelerometers method. 

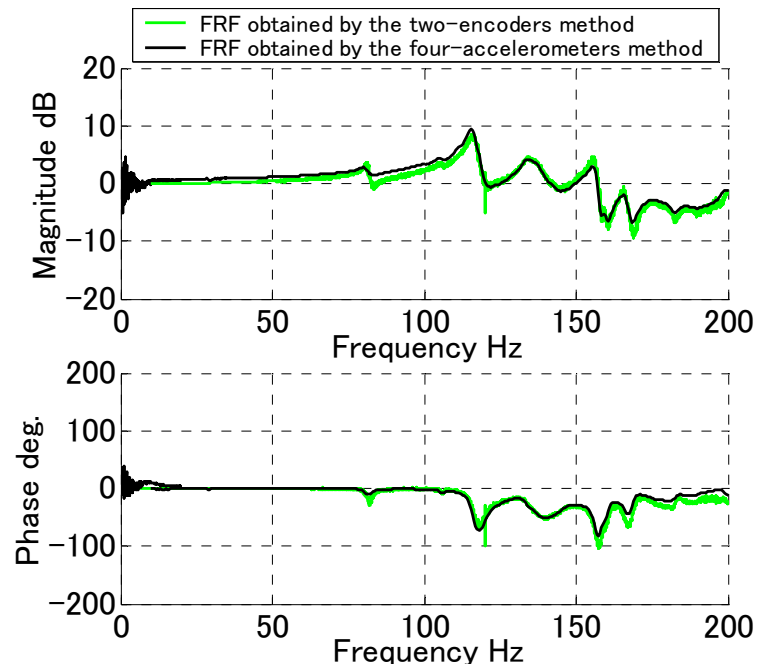

Fig. $5 G_{e t}(s)$ in the $\mathrm{X}$ direction measured by the four-accelerometers method and the two-encoders method. 

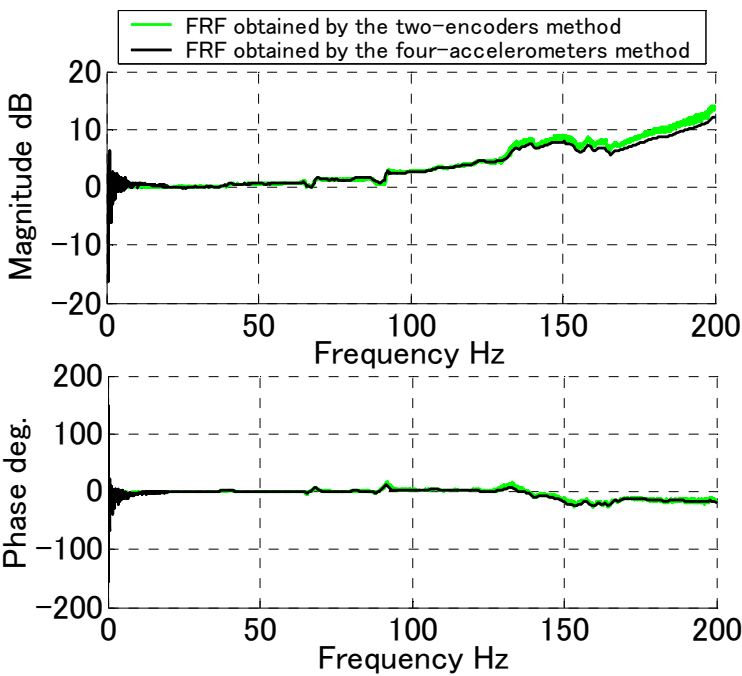

Fig. $6 G_{e t}(s)$ in the $\mathrm{Y}$ direction measured by the four-accelerometers method and the two-encoders method. 

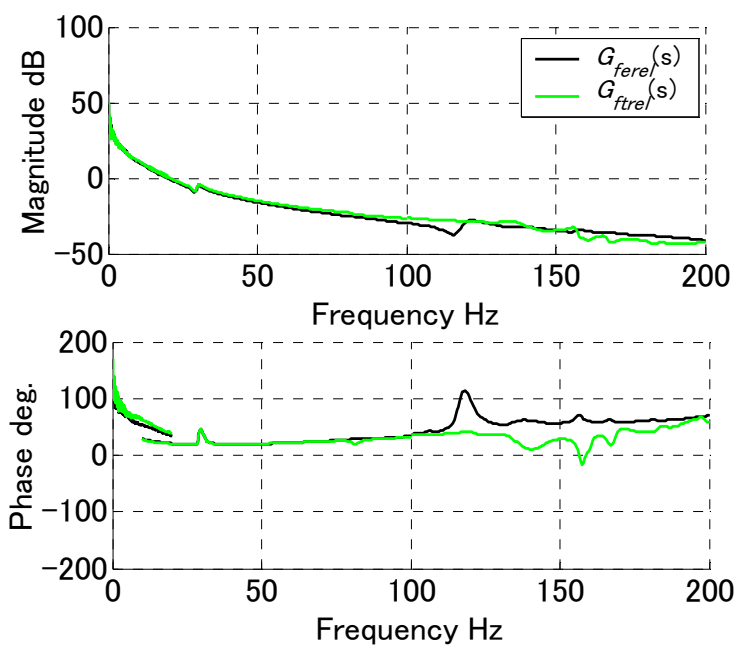

Fig. $7 G_{\text {ferel }}(s)$ and $G_{\text {frrel }}(s)$ in the X direction obtained by the four-accelerometers method. 

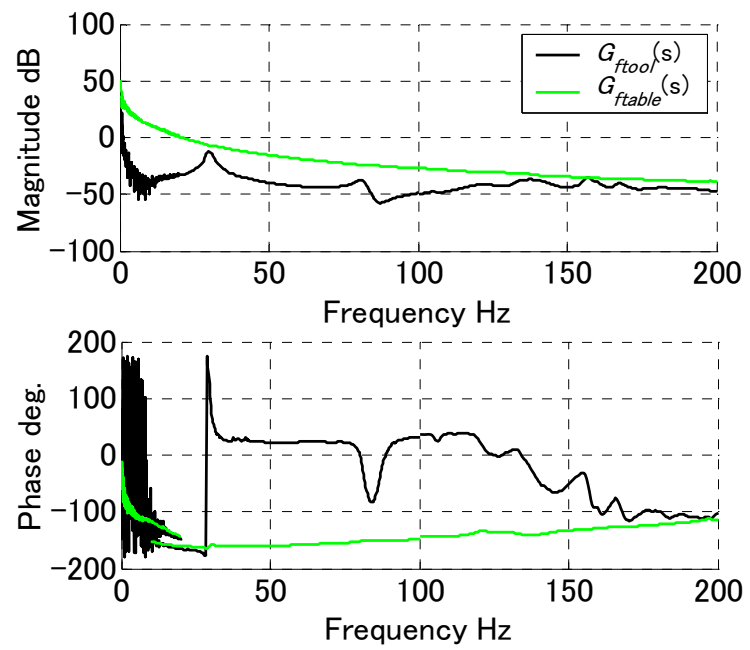

Fig. $8 G_{\text {ftool }}(s)$ and $G_{\text {ftable }}(s)$ in the X direction 

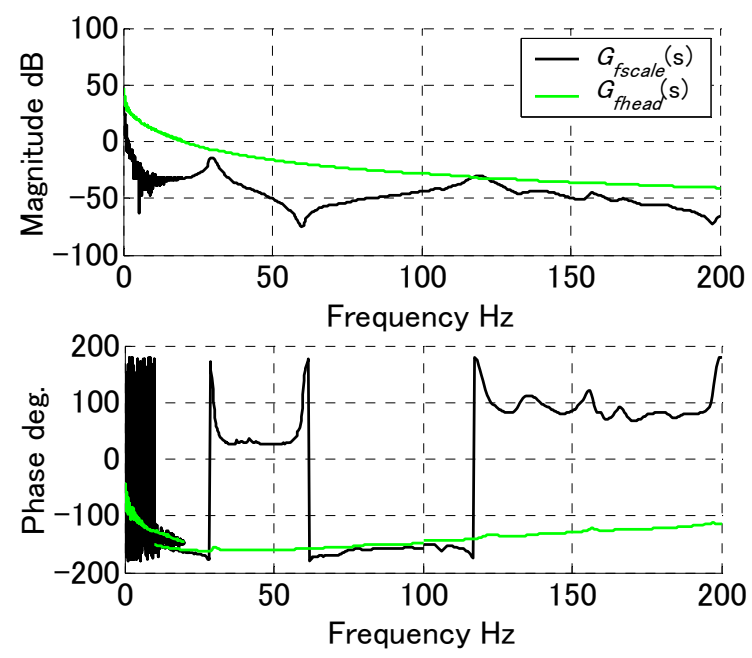

Fig. $9 G_{\text {fscale }}(s)$ and $G_{\text {fhead }}(s)$ in the X direction. 

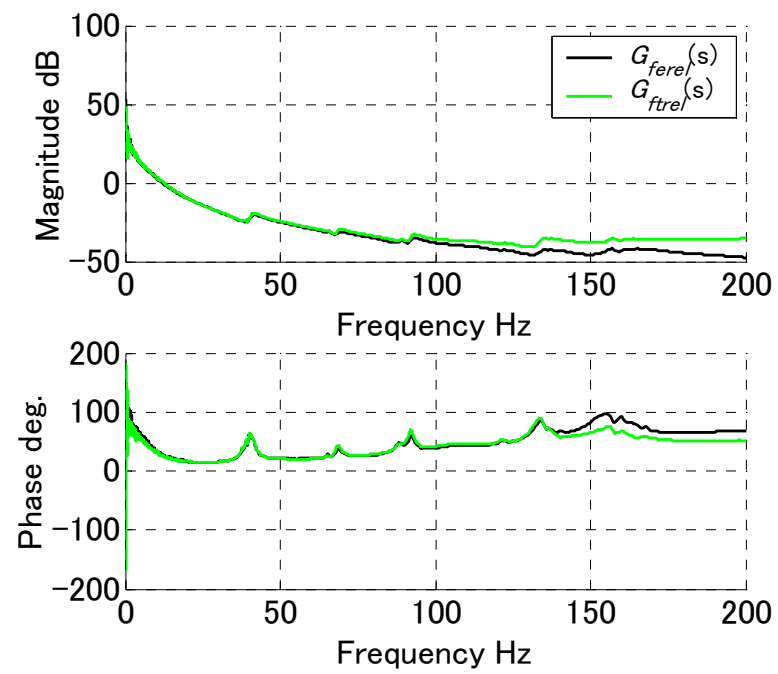

Fig. $10 G_{\text {ferel }}(s)$ and $G_{\text {frrel }}(s)$ in the Y direction obtained by the four-accelerometers method. 


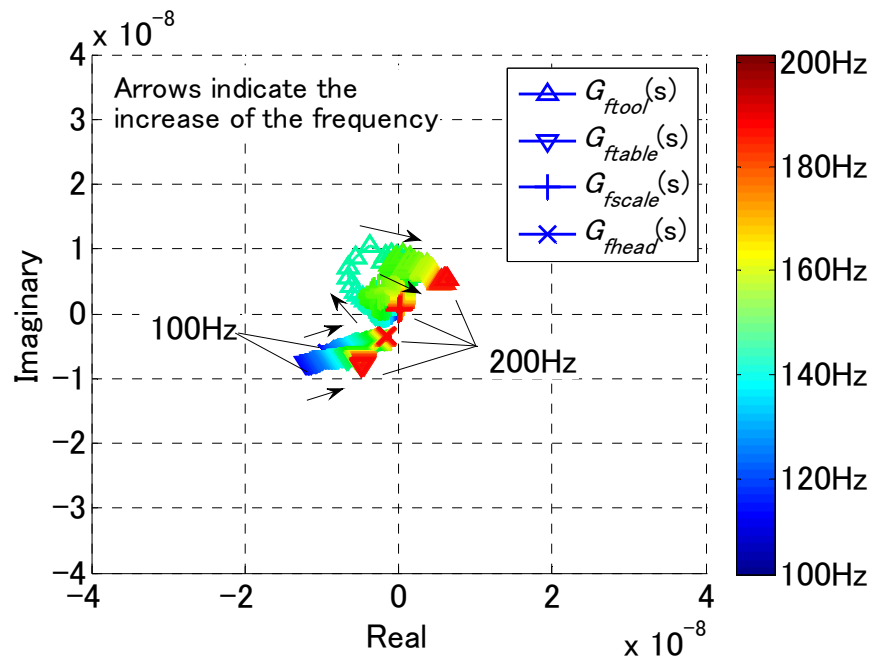

Fig. 11 Nyquist plot of $G_{\text {ftool }}(s), G_{\text {ftable }}(s), G_{\text {fscale }}(s)$, and $G_{\text {fhead }}(s)$ in the Y direction. 


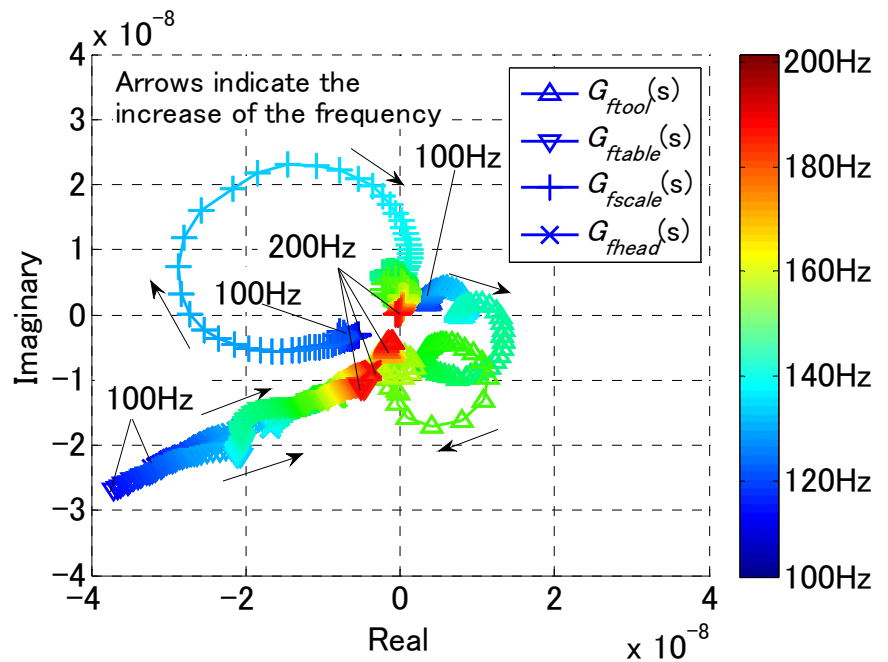

Fig. 12 Nyquist plot of $G_{\text {ftool }}(s), G_{\text {ftable }}(s), G_{\text {fscale }}(s)$, and $G_{\text {fhead }}(s)$ in the X direction. 


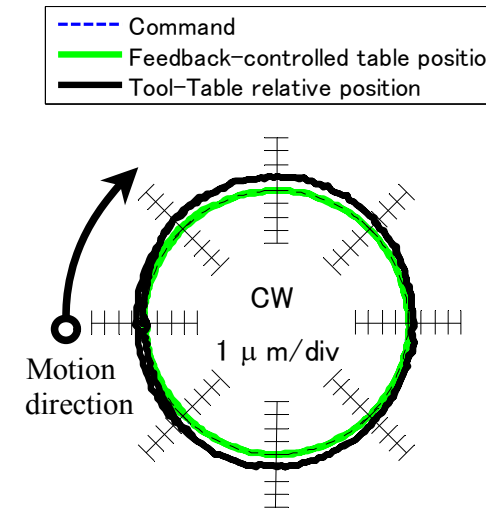

(a) Radius $=5 \mathrm{~mm}$

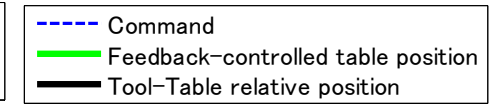

-----' Command

Feedback-controlled table position

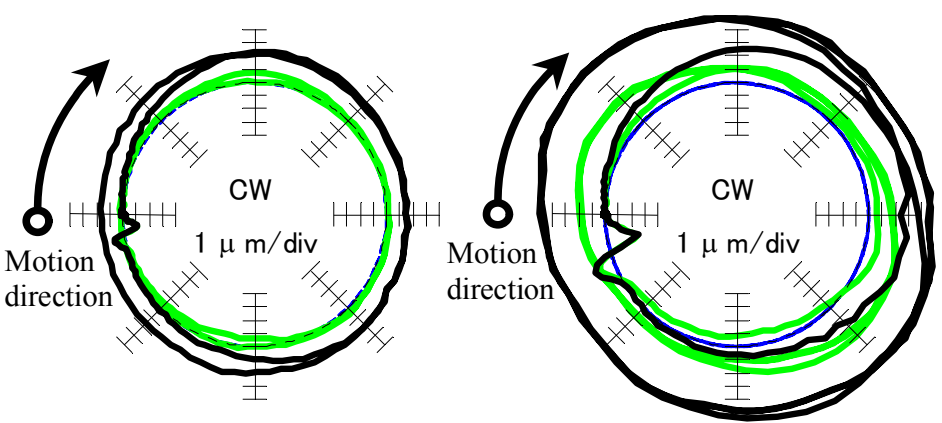

(b) Radius $=2 \mathrm{~mm}$

(c) Radius $=1 \mathrm{~mm}$

Fig. 13 Error contours for circular motion. Feed rate $=5 \mathrm{~m} / \mathrm{min}$. 


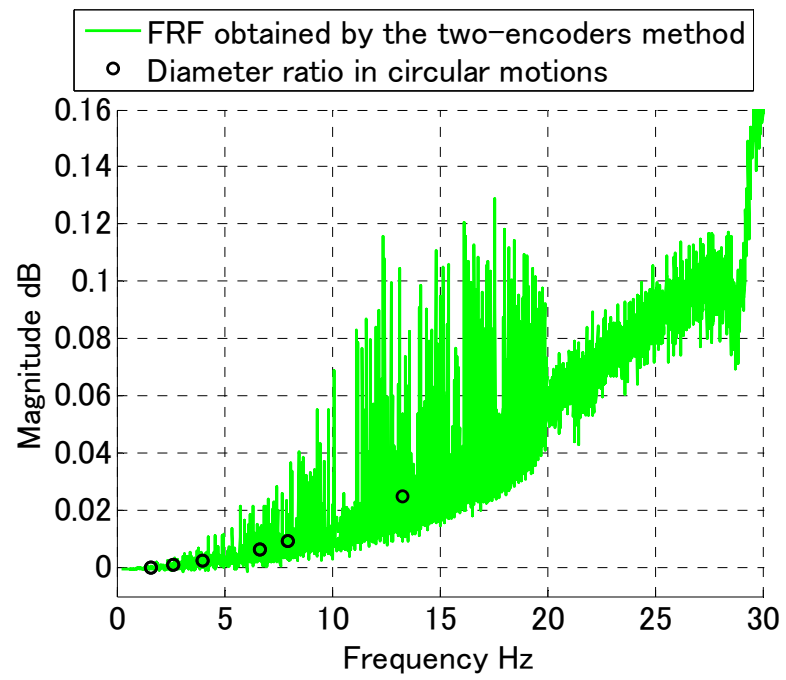

(a) Comparison in the $\mathrm{X}$ direction

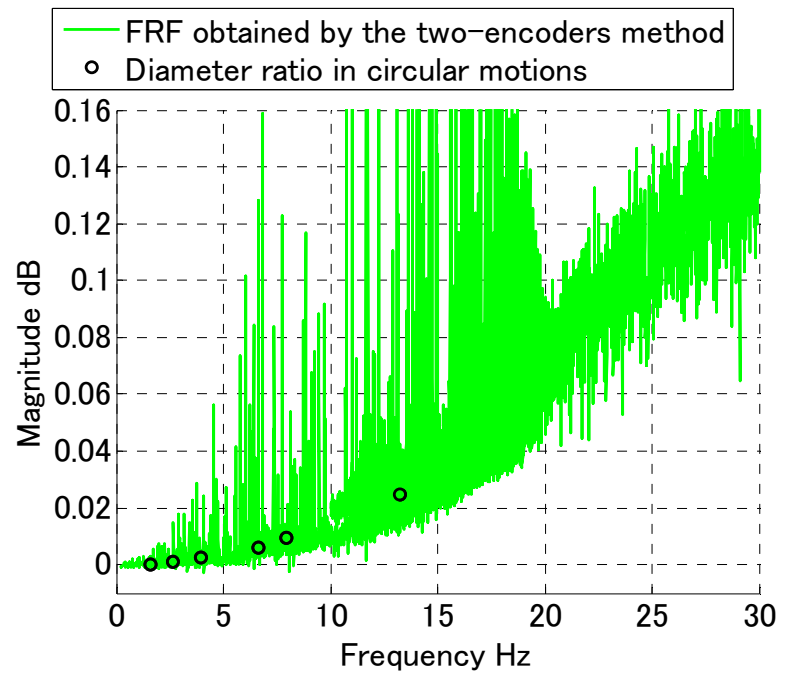

(b) Comparison in the $\mathrm{Y}$ direction

Fig. 14 Comparison between the magnitude of $G_{e t}(s)$ and the diameter ratio. 